\section{Gingival recession: part 1. Aetiology and non-surgical management}

\author{
M. Patel, ${ }^{1}$ P. J. Nixon ${ }^{2}$ and M. F. W.-Y. Chan ${ }^{3}$ \\ VERIFIABLE CPD PAPER
}

IN BRIEF
- Direct mechanical/physical influence
or indirect factors resulting in gingival
inflammation are key aetiological factors
in gingival recession.
- Treatment may only be necessary
for those patients presenting with
an aesthetic concern or dentine
hypersensitivity.
- Several surgical and non-surgical
treatment options are available.
(⿸丆口

Gingival recession is a common finding in many patients. Some patients will not be concerned whereas others will have aesthetic concerns or complain of sensitivity. This paper highlights the aetiology of gingival recession, the treatment options available to treat any associated sensitivity and the non-surgical treatment options available to restore aesthetics in patients with gingival recession. Subsequent papers in this series discuss the surgical treatment options available to correct localised recession defects.

\section{GINGIVAL RECESSION}

Gingival recession is the displacement of the gingival soft tissue margin apical to the cemento-enamel junction which results in exposure of the root surface. ${ }^{1}$ The prevalence of gingival recession has been shown to increase with age and can occur in patients with good standards of oral hygiene as well as those with poor oral hygiene and periodontal disease. ${ }^{2}$

\section{AETIOLOGY}

Gingival recession occurs either due to a direct mechanical or physical influence on the gingival tissues or indirectly due to an inflammatory reaction in the gingival tissues.

\section{Mechanical/physical factors}

These include aetiological factors which cause direct apical migration of the gingival tissues. These are:

a. Vigorous tooth brushing or by brushing with a hard bristle toothbrush are common causes of recession and this is often seen in patents with good oral hygiene. ${ }^{1-3}$ It usually presents as localised areas of recession mainly affecting the buccal surfaces of individual

\footnotetext{
"Specialist Registrar in Restorative Dentistry,

${ }^{2,3}$ Consultants in Restorative Dentistry, Department of Restorative Dentistry, Leeds Dental Institute, Clarendon Way, Leeds, LS2 9LU

*Correspondence to: Dr Mital Patel

Email: mitalpatel@nhs.net
}

\section{Refereed Paper}

Accepted 21 July 2011

DOI: 10.1038/sj.bdj.2011.764

${ }^{\circledR}$ British Dental Journal 2011; 211: 251-254

\begin{tabular}{|c|c|}
\hline Class & Description \\
\hline I & Mandibular incisors impinge on the palatal mucosa \\
\hline ॥ & $\begin{array}{l}\text { Mandibular incisors impinge on the palatal gingival margin of the maxillary incisors. Common } \\
\text { in Class II Div } 1 \text { relationships }\end{array}$ \\
\hline III & $\begin{array}{l}\text { Mandibular incisors impinge on the palatal gingival margin of the maxillary teeth and the } \\
\text { maxillary incisors impinge on the labial gingival margin of the mandibular incisors. Common in } \\
\text { Class II Div } 2 \text { incisal relationship }\end{array}$ \\
\hline IV & $\begin{array}{l}\text { Associated with the wear facets developing on the palatal surface of the maxillary incisors and/ } \\
\text { or labial surfaces of the mandibular teeth }\end{array}$ \\
\hline
\end{tabular}

teeth or a group of teeth and will have wedge shaped defects with minimal interproximal recession. For the majority of people the area of recession is more commonly associated with the left side of their mouth. This relates directly to the fact most people are right handed and brush the left side of their mouth first, when they are most effective. ${ }^{1}$ The gingival tissues often appear to be healthy around the area of recession and the exposed root surface is smooth, clean and polished. Buccal abrasion cavities may also be found with the area of recession

b. Traumatic incisal relationship can cause striping of the gingival tissues. Akerly $^{4}$ described four incisal relationships and their effect on the soft and hard tissues (Table 1). Akerly Class II and III highlight that a traumatic Class II Div 1 or Class II Div 2 incisal relationships can cause localised recession of the upper anterior teeth palatally and/or lower anterior teeth labially

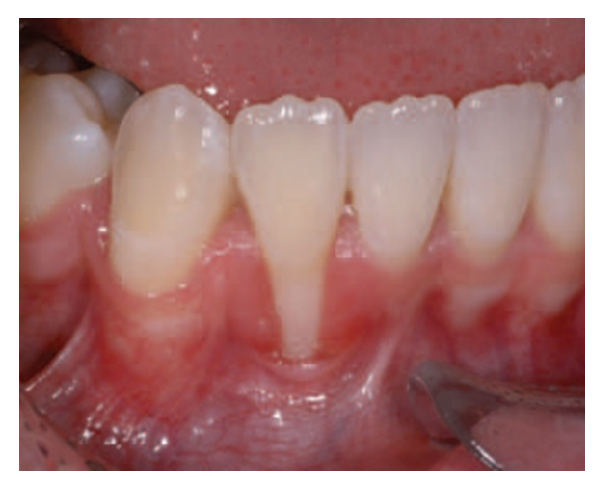

Fig. 1 Example of thin gingival biotype and recession associated with bone dehiscence

c. Trauma from foreign bodies such as lower lip piercings may also cause recession. ${ }^{5}$ Similarly, poorly designed tissue borne partial dentures can also result in gingival stripping leaving a recession defect

d. Teeth which are prominent and out of alignment of the arch maybe associated with alveolar dehiscence which can result in recession especially if there is thin gingival biotype (Fig. 1) overlying the dehiscence ${ }^{1,3,6}$ 
e. Aberrant fraenal attachments have been mentioned as a cause of recession due to an apical pull on the gingival tissues, however, the evidence for this is poor. ${ }^{1,6}$ High fraenal attachments (close to the gingival margin) may make oral hygiene difficult therefore leading to a localised periodontal problem and subsequent recession (Figs $2 \mathrm{a}$ and $2 \mathrm{~b}$ )

f. Recession can often occur as a result of any iatrogenic damage caused by restorative or periodontal treatment the patient may have. Restorative treatment which involves placement of subgingival margins of restorations can directly impinge on the biologic width. To reestablish the biologic width there may be some bone loss and apical migration of the gingival tissues. ${ }^{2}$

Successful treatment of periodontal disease and gingivitis will also result in the apical movement of the gingival margin. In some cases where there are shallow periodontal pockets, repeated root planing can also result in resorption of the crestal bone and gingival recession.

\section{Gingival recession caused by an inflammatory process}

There are various predisposing factors which can result in recession due to inflammation of the gingival tissues. These include:

a. Gingival biotype. The height of keratinised tissue is not an important factor in predicting recession; however, evidence shows the thickness of the keratinised tissue is an important prognostic factor. Subgingival plaque results in presence of inflammation around the gingival margin. This area of inflammation rarely extends more than 1-2 mm apically and laterally therefore where the free gingival tissue is thick only a small area of connective tissue is affected. However, where the free gingival tissue is thin and delicate or in an area of alveolar dehiscence the entire connective tissue can be affected resulting in recession. ${ }^{2}$ Figure 1 shows an example of thin gingival biotype and Figure 3 shows an example of thick gingival biotype

b. Periodontal disease is another common cause of recession which results in the loss of the supporting bone around a tooth. ${ }^{1}$ Usually a tooth affected by periodontal disease will lose bone support around the tooth through an inflammatory reaction which results in apical migration of the soft tissue margin. These patients are likely to show generalised signs of recession on all surfaces of the teeth (interproximal, buccal and lingual/ palatal), ${ }^{2}$ although there are exceptions

c. Poor marginal fit, inadequate crown emergence angles, rough restoration surfaces and overhangs on restorations can result in a plaque trap. This can cause gingival inflammation if the patient is not meticulous with their oral hygiene and subsequently lead to recession of the gingival tissues ${ }^{2}$

d. Orthodontic tooth movement per se will not cause recession; however, orthodontic movement of teeth labially outside the envelope of alveolar bone will result in loss of buccal bone (alveolar dehiscence) and a decrease in gingival tissue thickness due to stretching of the gingival tissue fibres. ${ }^{1,2}$ The decreased thickness of gingival tissue mimics a thin gingival biotype which as discussed above is more prone to recession from plaque induced inflammation or even tooth brushing trauma. If a tooth is moved lingually or palatally within the envelope of the alveolar process, there is reduced risk of recession defects developing as there is no pressure and stretching of the labial gingival tissue and therefore it maintains its protective function against plaque induced inflammation.

\section{PATIENT COMPLAINTS/CONCERNS}

Gingival recession is a common feature seen in many patients. Some patients will be unaware of the condition, others will be aware of it but not concerned about it whereas some will be concerned about it and will want it corrected. Patients tend to present with three main concerns, which are poor aesthetics, worry about potential tooth loss and dentine hypersensitivity due to the exposed root surface following gingival recession. ${ }^{7}$ Recession may also be associated with cervical lesions such as abrasive class $\mathrm{V}$ cavities or root caries.

\section{GINGIVAL AESTHETICS}

In order to treat recession and address the patient's aesthetic concerns it is important to have an understanding of gingival

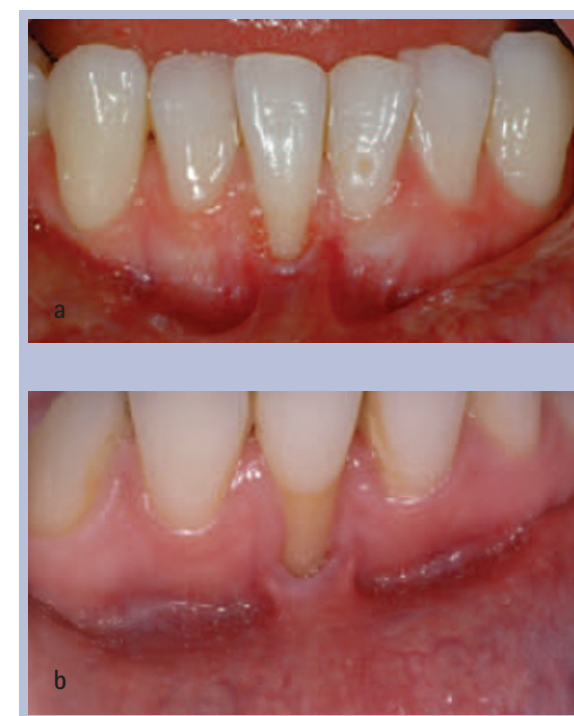

Figs 2a-b Examples of localised recession associated with high fraenal attachment

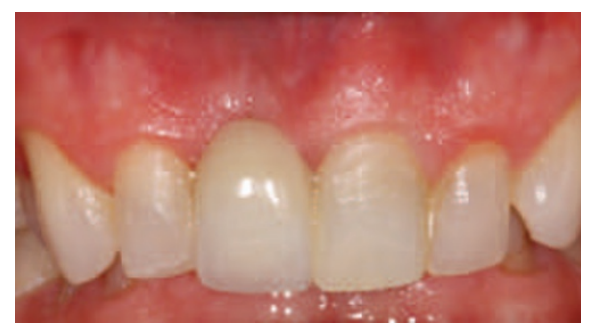

Fig. 3 Example of thick gingival biotype which is least prone to recession

aesthetics and the lip line. When considering aesthetics we must first assess the patient's lip line. This should be done at rest, while the patient is talking and when the patient smiles to show the highest level of the lip line. The key features to note are the symmetry in the smile, the amount of tooth tissue visible and the amount of gingival show. Once an extra oral assessment of the aesthetics has been completed then a closer look at the oral tissues can be carried out. This involves initially looking at the teeth to see which teeth are present, their position in the arch and the symmetry between the two sides, followed by an assessment of the gingival tissues.

In the dental literature several authors have described the ideal position of the gingival zenith (the highest point of the gingival margin around a tooth) relative to the vertical midline of the tooth. ${ }^{8-10} \mathrm{Chu}$ et al..$^{10}$ carried out a study looking at 20 healthy patients with the average age of 27.7 years and found that on average the gingival zenith is $1 \mathrm{~mm}$ distal to the midline on the central incisors, $0.4 \mathrm{~mm}$ distal to the midline on the lateral incisors and the canine 


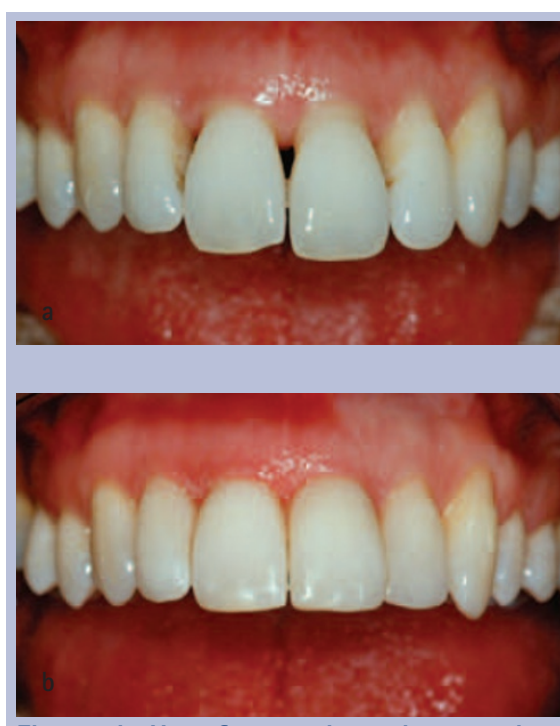

Figs 4a-b Use of composite resin to mask recession defects and eliminate black triangles caused by recession. Enameloplasty was carried out to even incisal plane

had no deviation from the midline. Other authors have suggested that the zenith of the central incisor is as described above; the lateral incisors have the zenith along the midline and the canines slightly distal to the midline. ${ }^{9,11}$ In reality the true position of the zenith may not be important as long as there is symmetry. It is important for us to understand these aesthetic dimensions when assessing the patient's aesthetics, the amount of recession and for planning treatment to correct the recession.

\section{TREATMENT}

The aim of the treatment for gingival recession should be to address the patient's concerns of sensitivity and/or aesthetics. The treatment options available include:

1. Monitoring and prevention

2. Use of desensitising agents, varnishes and dentine bonding agents

3. Composite restoration

4. Pink porcelain or composite

5. Removable gingival veneers

6. Orthodontics

7. Surgery.

\section{NON-SURGICAL}

TREATMENT OPTIONS

\section{Monitoring and prevention of further recession}

If the recession defect is minimal, not in the aesthetic zone and there is no associated dentine hypersensitivity or root caries it may be acceptable to the patient to do nothing. In these cases it is important to identify and manage the cause of recession such as tooth brushing trauma or chronic periodontal disease to prevent further recession. It is also important to maintain good oral hygiene to prevent further plaque induced inflammation which can result in further recession especially in thin gingival biotypes.

In cases where the patient has a high smile line and the gingival zeniths are uneven due to recession or where root caries or dentine hypersensitivity develops the patient may seek treatment.

\section{Desensitising agents, varnishes and dentine bonding agents to treat dentine hypersensitivity}

If the patient's main complaint is sensitivity and aesthetics are not a concern, then concentrating efforts to treat the sensitivity alone may be enough. Patients suffering from dentine hypersensitivity may avoid brushing areas which are sensitive which can result in accumulation of plaque and further plaque induced recession. It is therefore important to treat sensitivity. Treatment of dentine hypersensitivity is based on either blocking the dentinal tubules or preventing nerve stimulation. There are many products available which aim to cover the root surface and block the dentinal tubules to prevent fluid movement. Examples of these products include varnishes, dentine bonding agents, glass ionomer cements or composite resins. The more resistant the product is to its removal off the root surface, the better it will be at treating sensitivity. Toothpastes and mouthwashes are also widely available in the market to help treat sensitivity. Some products containing either strontium or potassium aim to stabilise the nerve by decreasing the nerve excitability. However, a recent systematic review has suggested that the evidence for this is weak. ${ }^{12}$ Other products available contain silica and oxalates which aim to occlude the dentinal tubules. The literature on treatment of dentine hypersensitivity does not show one treatment modality being better than the others. If toothpastes are used, there may be some benefit in smearing the toothpaste over the exposed root surface and leaving it for approximately 30 minutes before brushing or rinsing it away. This would leave the active agents within the toothpaste in contact with the root surface for longer giving them more of a chance at reducing the sensitivity. More recently lasers have also been advocated for the treatment of dentine hypersensitivity. They are thought to cause coagulation of proteins in the dentinal tubules which decreases permeability and allows development of an amorphous sealed layer of dentine caused by meltdown of the surface but there is a lack of long term evidence for this. ${ }^{13,14}$

\section{Composite restorations}

With the advancement in composite resin materials and dentine bonding techniques, adhesive composite restorations are becoming more and more popular and predictable in restorative dentistry. Small localised recession defects with sensitivity, wear or caries of the root surface can be corrected by bonding tooth coloured composite over the exposed root surface. This will result in a longer clinical crown height which would be acceptable if the patient has a low lip line or if adjacent teeth can be treated in the same way to maintain symmetry. On some occasions it may also be possible to use composite to treat more extensive recession defects where there has been interproximal bone loss often seen in patients with periodontal disease. The composite can be used to close the black triangles and cover the exposed root surfaces associated with the recession defect (Figs 4a-b). Careful placement of the composite restoration is essential to ensure there are no plaque retentive margins which would promote further gingival recession. In some cases using tooth coloured composite in this way may not be aesthetically acceptable and alternative options would need to be considered to restore the aesthetics.

\section{Pink porcelain or composite}

Surgical procedures have been widely described to successfully treat recession defects; however, in some patients surgery may not be a viable option or an option they wish to pursue. With advances in bonding agents and the development of pink ceramics and resin composite materials, it is possible to use gingival coloured porcelain or composites over the root surface to eliminate dentine hypersensitivity 
and restore aesthetics. ${ }^{7}$ However, there are some difficulties associated with these techniques such as getting a good colour match of the restorative material with the gingival tissues, moisture control from the gingival crevicular fluid and ensuring there are no ledges as it can make oral hygiene difficult leading to further recession.

\section{Removable gingival veneers}

Some patients will have multiple sites of recession in the anterior aesthetic zone which is most commonly associated with periodontal disease. This results in exposed root surfaces and appearance of spacing between the teeth where the dental papillae have been lost, often referred to as 'black triangles'. Some patients will also complain of altered speech due to air escaping through the recession defects interproximaly. In these patients surgical techniques to graft multiple sites to replace lost tissue may be impossible and unpredictable.

The use of a removable gingival prosthesis can replace large volumes of receded soft tissue, fill the interproximal spaces to eliminate the black triangles and improve aesthetics (Figs 5a-c). Removable gingival prostheses can be made from various materials which include heat cured acrylic or silicone based soft lining materials such as molloplast B. ${ }^{15,16}$ A study by Lai et al. ${ }^{17}$ showed that gingival veneers made from heat cured acrylic had more colour stability compared to silicone or co-polyamide materials which were prone to staining from tea and coffee. On the other hand acrylic materials have the disadvantage of being hard, ridged, easy to fracture and difficult to fit around teeth. In comparison silicone base materials are flexible, have improved comfort and increased resistance to fracture.

The gingival prosthesis is made from a two part impression taken in silicone (Fig. 6). Silicone putty is initially placed on the palatal aspect ensuring it does not go past the maximum curvature of the teeth within the interproximal spaces. A separating medium such as petroleum jelly is placed on the palatal impression once set and re-seated in the mouth. The labial portion of the impression can then be taken with a heavy or medium bodied silicone using a special custom made buccal impression tray or using silicone putty as shown in Figure 6.

\section{Orthodontics}

Teeth which may be malpositioned buccally/labially through development may have a buccal dehiscence and associated recession as discussed previously. This is often seen in buccally placed lower incisor teeth where there is crowding of the lower labial segment. In some cases surgical intervention and grafting may help to treat the recession defect; however, if orthodontic treatment is an option that the patient is willing to consider then any surgical intervention should be delayed until after orthodontic tooth movement has been completed. Studies have shown that orthodontic movement of the tooth lingually allows alveolar bone growth on the buccal aspect, thickening of the gingival tissue and subsequent coronal shift in the gingival margin resulting in correction of the recession defect. If following orthodontic treatment surgical intervention is still indicated the outcome is likely to have higher predictability than if it was performed before orthodontic treatment. ${ }^{2,18}$

\section{CONCLUSION}

Gingival recession is often seen in the patients presenting to the general dental practitioner or restorative specialist. Many of these patients can often be treated simply by employing the non-surgical techniques discussed above. In some cases surgical intervention may be necessary and subsequent papers in this series aim to discuss the surgical management of recession.

1. Kassab M M, Cohen R E. The etiology and prevalence of gingival recession. J Am Dent Assoc 2003; 134: 220-225.

2. Baker $P$, Spedding $C$. The aetiology of gingival recession. Dent Update 2002 : 29: 59-62.

3. Gorman W J. Prevalence and etiology of gingival recession. J Periodontol 1967; 38: 316-322.

4. Akerly W B. Prosthodontic treatment of traumatic overlap of the anterior teeth. J Prosthet Dent 1977; 38: 26-34.

5. Er N, Ozkavaf A, Berberoglu A, Yamalik N. An unusual cause of gingival recession: oral piercing. J Periodontol 2000; 71: 1767-1769.

6. Wennstrom J L. Mucogingival therapy. Ann Periodontol 1996: 1: 671-701.

7. Zalkind M, Hochman N. Alternative method of conservative esthetic treatment for gingival recession. J Prosthet Dent 1997; 77: 561-563.

8. Reddy M S. Achieving gingival esthetics. J Am Dent Assoc 2003; 134: 295-304.

9. Gill D S, Naini F B, Tredwin C J. Smile aesthetics. Dent Update 2007: 34: 152-154, 157-158.

10. Chu S J, Tan J H, Stappert C F, Tarnow D P. Gingival zenith positions and levels of the maxillary anterior dentition. J Esthet Restor Dent 2009; 21: 113-120, discussion 21

11. Davis N C. Smile design. Dent Clin North Am 2007; 51: 299-318, vii.

12. Poulsen S, Errboe M, Lescay Mevil Y, Glenny A M.
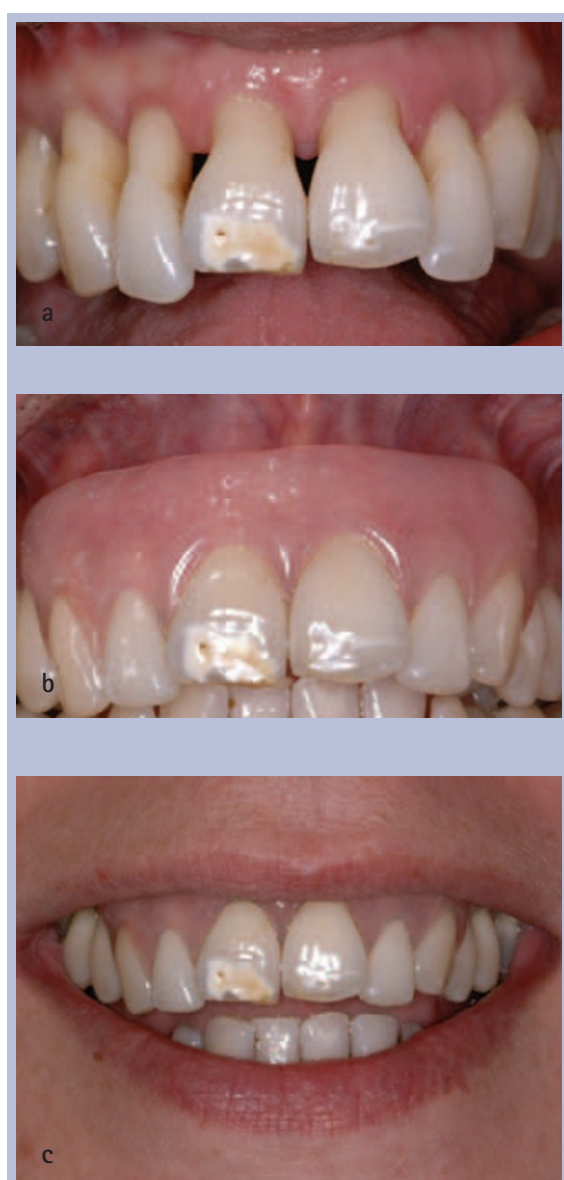

Figs 5a-c Gingival veneer used to correct recession caused by periodontal disease

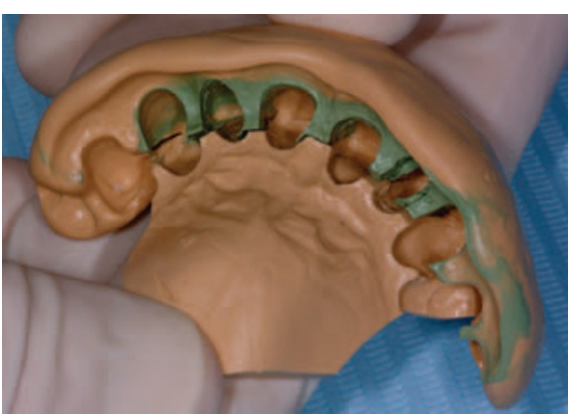

Fig. 6 Two part impression technique for gingival veneer

Potassium containing toothpastes for dentine hypersensitivity. Cochrane Database Syst Rev 2006; (3): CD001476.

13. West N X. Dentine hypersensitivity: preventive and therapeutic approaches to treatment. Periodontol 2000 2008; 48: 31-41.

14. Al-Sabbagh M, Brown A, Thomas M. In-office treatment of dentine hypersensitivity. Dent Clin North Am 2009; 53: 47-60.

15. Barzilay I, Irene T. Gingival prostheses - a review. J Can Dent Assoc 2003; 69: 74-78

16. Carvalho W, Barboza E P, Gouvea C V. The use of porcelain laminate veneers and a removable gingival prosthesis for a periodontally compromised patient: a clinical report. J Prosthet Dent 2005; 93: 315-317.

17. Lai $Y$ L, Lui H F, Lee S Y. In vitro color stability, stain resistance, and water sorption of four removable gingival flange materials. J Prosthet Dent 2003; 90: 293-300.

18. Wennstrom J L. Mucogingival considerations in orthodontic treatment. Semin Orthod 1996; 2: 46-54 